\title{
Serum Daytime Melatonin Levels Reflect Cerebrospinal Fluid Melatonin Levels in Alzheimer's Disease but Are Not Correlated with Cognitive Decline
}

\author{
Amber Nous $^{\mathrm{a}, \mathrm{b}, \mathrm{c}}$, Mandy Melissa Jane Wittens ${ }^{\mathrm{a}, \mathrm{b}, \mathrm{c}}$, Yannick Vermeiren ${ }^{\mathrm{c}, \mathrm{d}, \mathrm{e}}$, \\ Peter Paul De Deyn ${ }^{\mathrm{c}, \mathrm{f}}$, Christine Van Broeckhoven ${ }^{\mathrm{c}, \mathrm{g}, \mathrm{h}}$, Guy Nagels ${ }^{\mathrm{a}, \mathrm{b}}$, \\ Ilse Smolders ${ }^{\mathrm{b}, 1, *}$ and Sebastiaan Engelborghs $\mathrm{s}^{\mathrm{a}, \mathrm{b}, \mathrm{c}, 1, *}$ \\ ${ }^{a}$ Department of Neurology, Universitair Ziekenhuis Brussel (UZ Brussel), Brussels, Belgium \\ ${ }^{\mathrm{b}}$ Center for Neurosciences, Vrije Universiteit Brussel, Brussels, Belgium \\ ${ }^{\mathrm{c}}$ Department of Biomedical Sciences, University of Antwerp, Antwerp, Belgium \\ ${ }^{\mathrm{d}}$ Faculty of Medicine \& Health Sciences, Translational Neurosciences, University of Antwerp, Antwerp, Belgium \\ ${ }^{\mathrm{e}}$ Division of Human Nutrition and Health, Chair Group of Nutritional Biology, Wageningen University and \\ Research, Wageningen, Netherlands \\ ${ }^{\mathrm{f}}$ Department of Neurology and Memory Clinic, Hospital Network Antwerp (ZNA) Middelheim and Hoge Beuken, \\ Antwerp, Belgium

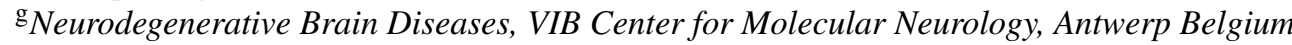

Handling Associate Editor: Piotr Lewczuk

Accepted 23 June 2021

Pre-press 2 August 2021

\begin{abstract}
. in $\mathrm{AD}$ patients still has to be undertaken. observed between daytime melatonin levels and MMSE score changes.

\footnotetext{
${ }^{1}$ These authors are joint last authors.

*Correspondence to: Ilse Smolders and Sebastiaan Engelborghs, Vrije Universiteit Brussel, Center for Neurosciences, Laarbeeklaan 103, 1090 Jette, Belgium. Tel.: +32 247764 10; E-mails: Ilse.Smolders@vub.be, Sebastiaan.Engelborghs@vub.be
}

Background: Nocturnal cerebrospinal fluid (CSF) and blood melatonin levels are altered in Alzheimer's disease (AD). However, literature remains inconclusive on daytime blood melatonin levels. A positive correlation between melatonin levels and Mini-Mental State Examination (MMSE) scores in AD subjects has been evidenced following cross-sectional analyses. Whereas a correlation between serum and spinal CSF melatonin has been shown in healthy volunteers, an equal investigation

Objective: 1) To evaluate whether serum melatonin levels correlate with spinal CSF melatonin levels in AD. 2) To compare daytime CSF and serum melatonin levels between patients with $\mathrm{AD}$ dementia, mild cognitive impairment due to $\mathrm{AD}$, and healthy controls, and to evaluate whether melatonin can affect cognitive decline in AD.

Methods: Subjects with AD and healthy controls included in two existing cohorts, of whom a CSF and serum sample was available at the neurobiobank and had at least 6 months of neuropsychological follow-up, were included in the present study. Melatonin concentrations were measured with liquid chromatography-mass spectrometry.

Results: Daytime serum melatonin levels correlated with spinal CSF melatonin levels in $\operatorname{AD}(r=0.751, p<0.001)$. No significant differences regarding daytime melatonin levels were found between patients and controls. No correlations were 
Conclusion: Daytime serum melatonin accurately reflects CSF melatonin levels in AD, raising the possibility to assess melatonin alterations by solely performing blood sampling if also confirmed for night-time values. However, daytime melatonin levels are not associated with changes of cognitive impairment.

Keywords: Alzheimer's disease, cerebrospinal fluid, dementia, melatonin

\section{INTRODUCTION}

Melatonin is a neurohormone produced by the pineal gland under influence of the hypothalamic suprachiasmatic nucleus ( $\mathrm{SCN}$ ) and is part of our circadian rhythm, with low levels during the day $(<5 \mathrm{pg} / \mathrm{ml}$ in plasma [1]) and high levels during the night in healthy adults (average $60 \mathrm{pg} / \mathrm{ml}$ in serum [2] [3-5]). Melatonin produced in the pineal gland reaches the third ventricle directly via the pineal recess and might furthermore be produced by glial cells such as astrocytes, leading to high melatonin levels in cerebrospinal fluid (CSF) $(32.5 \mathrm{pg} / \mathrm{ml}$ for daytime CSF melatonin collected from lumbar cistern $[6,7])$. On the other hand, melatonin enters the blood stream through passive diffusion when the synthesis of melatonin increases at night [8].

Melatonin production is disrupted in Alzheimer's disease (AD), the most common cause of dementia [9-16]. A loss of volume and cells of the SCN when comparing $\mathrm{AD}$ patients with age-matched controls has been described, as well as decreased functional pineal volumes [17-22]. Furthermore, a loss of melatonin rhythmicity in postmortem pineal glands with disappearance of the significant increase in melatonin levels in those $\mathrm{AD}$ patients who died at night versus those who died during the day, and a significant reduction in pineal night-time melatonin levels in $\mathrm{AD}$ patients as compared to controls has been shown [10, 23]. Ventricular CSF melatonin levels of clinically and pathologically confirmed $\mathrm{AD}$ patients were found to be one fifth of age-matched healthy controls [9]. Several studies have been performed regarding blood melatonin levels in $\mathrm{AD}$, showing a loss in diurnal variations [12, 13, 24, 25], decreased total levels of melatonin [15], decreased nocturnal peaks [11, 12, $15,24,25]$, and earlier onset of the nocturnal melatonin rise [12] or wider scatter of the acrophase [11]. However, increased [12,13] as well as decreased [14, $26,27]$ or unaltered $[11,15,28]$ daytime blood melatonin levels have been reported in patients with $\mathrm{AD}$ as compared to healthy controls. In addition, the effect of $A P O E \varepsilon 4$ carrier status on melatonin levels in CSF and blood of $\mathrm{AD}$ patients remains unclear [9, 28, 29].
Aberrant melatonin production might contribute to sleep disruptions in $\mathrm{AD}$, including nightly restlessness and sundowning [4]. This might lead to disease progression due to decreased night-time functioning of the glymphatic system, which according to the glymphatic hypothesis $[30,31]$, is responsible for drainage of solutes like amyloid- $\beta(A \beta)$, or by a relative increase in neuronal activity with increased production of $A \beta$ as a consequence [32]. Melatonin has positive effects on sleep and cognition in mild cognitive impairment (MCI) patients, diagnosed according to the Petersen criteria [33, 34], but not in patients with dementia due to AD (ADD) where it only had positive effects on sleep quality [35]. Correlations between daytime as well as nocturnal blood melatonin levels and Mini-Mental State Examination (MMSE) scores or severity of dementia have been found [11, 14], but have not yet been studied in CSF, nor have they been correlated with changes in melatonin levels in a longitudinal way.

By direct secretion of melatonin in the third ventricle via the pineal recess, concentrations in (ventricular) CSF are higher than in the peripheral blood circulation [7]. A study in 13 healthy humans showed a correlation between spinal CSF melatonin levels and serum melatonin levels [6]. However, a more recent study showed that sleep deprivation in healthy subjects led to increased CSF and serum melatonin levels but with lack of correlation between both [36]. Whereas a very strong positive correlation between CSF melatonin levels and pineal melatonin levels in $\mathrm{AD}$ patients has been found [23], this has not yet been studied for blood and spinal CSF.

The aim of this retrospective study was to evaluate whether serum (daytime) melatonin levels accurately correlate with spinal CSF melatonin levels in the AD continuum. Furthermore, since current literature on daytime melatonin levels in $\mathrm{AD}$ produced conflicting evidence, we aimed to compare CSF and serum melatonin concentrations between patients with ADD, MCI due to $\mathrm{AD}$ (abbreviated as MCI as from here on), and healthy controls, in order to increase the usability of melatonin as blood-based biomarker. Lastly, we 
aimed to investigate whether these daytime melatonin levels are associated with cognitive decline in $\mathrm{AD}$.

\section{METHODS}

\section{Subjects}

Patients with dementia, patients with MCI, and healthy controls were included in a prospective, longitudinal observational study at the memory clinic of Hospital Network Antwerp (ZNA) Middelheim and Hoge Beuken. Patients were recruited between 1995 and 2013, cognitively healthy controls between 2014 and 2017 [37-39]. The protocol was approved by the local Ethics Committee (UZA/UAntwerp and ZNA), approval numbers 2805 and 2806. All participants and/or their legal representatives gave a written informed consent. Behavioral assessment and neuropsychological testing at baseline and during follow-up visits, as well as CSF and blood sampling for $\mathrm{AD}$ biomarker analysis and sample storage was done in some, but not all participants in the prospective study. For the current study, we retrospectively selected patients and controls from the Neurobiobank of the Institute Born-Bunge (NBB-IBB; FAGG registration $n^{\circ} 190113$ ) according to following diagnostic criteria and selection procedures.

\section{Diagnostic criteria}

We selected patients with a diagnosis of MCI and ADD based on the National Institute on Ageing and Alzheimer's disease (NIA-AA) criteria [40, 41], implying that diagnosis was supported by the core AD CSF biomarkers $\left(A \beta_{1-42}\right.$, total tau (T-tau) and

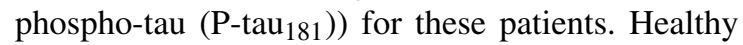
controls had normal CSF $A \beta_{1-42}$ values.

\section{Selection procedure}

\section{Patients}

All MCI and ADD patients of whom CSF and blood samples were available in the NBB-IBB 1) and in whom baseline neuropsychological testing was done within 3 months of CSF and blood sampling (before or after); 2) and who had neuropsychological testing done during follow-up visit at least 6 months after the first neuropsychological testing; 3 ) were included in the current research protocol. This resulted in the inclusion of 13 patients with $\mathrm{MCI}$ and 23 ADD patients in the current study.

\section{Cognitively healthy controls}

The 5 oldest healthy controls, best age-matched for the patient cohort, of whom CSF and blood samples were still available in the NBB-IBB 1) and in whom baseline neuropsychological testing was done within 4 months of CSF and blood sampling (before or after); 2) and who had neuropsychological testing done during follow-up visit at least 6 months after the first neuropsychological testing; 3) were included in the current research protocol.

None of the participants took beta- or alpha-blocking therapy, nor did they take melatonin treatment. However, information about medication intake was missing in 3 healthy controls.

\section{Procedures}

\section{Behavioral assessment}

Behavioral assessment was performed using a battery of behavioral assessment scales (CMAI [Cohen-Mansfield Agitation Inventory], BEHAVEAD [Behavioral pathology in Alzheimer's disease], MFS [Middelheim Frontality Score], GDeprS [30 item Geriatric Depression Scale], CSDD [Cornell Scale for Depression and Dementia], GDS [Global Deterioration Scale]) at baseline and during followup visits in patients as described earlier [37-39]. For controls, no behavioral assessment was done. However, we knew that healthy controls did not have significant depressive complaints.

For the current study, we focused on the BEHAVEAD E cluster which evaluates day/night rhythm disturbances, with a score ranging from 0 to 3 with 0 having no day/night disturbance and 3 meaning complete reversal of day/night rhythm $(<50 \%$ of former sleep cycle at night). Furthermore, we took into account the CSDD and GDeprS scores to evaluate the possible association of depression with melatonin levels. The CSDD is a 19-item depression scale. Item scores range from 0 (absent) to 2 (severe), with a maximum total score of 38 points. The items are clustered in five groups: (A) mood-related signs: anxiety, sadness, and lack of reactivity to pleasant events; (B) behavioral disturbances: agitation, retardation, multiple physical complaints, and loss of interest; (C) physical signs: appetite loss, weight loss, and lack of energy; (D) cyclic functions: diurnal variation of mood, difficulty falling asleep, multiple awakenings during sleep and early morning awakening; (E) ideational disturbances: suicide, poor self-esteem, pessimism, and mood-congruent delusions [37, 42]. The GDeprS is a 30 -item questionnaire in which 
participants are asked to respond by answering yes or no in reference to how they felt over the past week and is used as an instrument to detect and evaluate significant depressive symptoms [43]. Although the GDeprS was developed for cognitively healthy elderly, this scale remains valid in patients with MCI [44].

\section{Neuropsychological testing}

Neuropsychological testing was performed at baseline and during follow-up visits up to 3 years after baseline. Neuropsychological testing consisted of MMSE, and a battery of neuropsychological tests as routinely used for diagnosis and evaluation of dementia [37-39].

In the present study we focused on MMSE scores and MMSE score changes over a certain time period in order to evaluate cognitive decline. We assessed cognitive decline by evaluating [(last MMSE score measured - MMSE score baseline) / years between both] and defined this as "yearly MMSE score change".

\section{Lumbar puncture $(L P)$ procedure and}

blood sampling and pre-analytical sample handling

At baseline, a subgroup of patients and controls in the prospective research protocol underwent LP and blood sampling at the same time during daytime (between 08:00 AM and 04:00 PM, 78\% of samples were taken between 08:00AM and 10:00AM). LP and blood sampling was performed in normal hospital lighting conditions throughout all seasons of the year. Blood was taken at the same time of LP via peripheral venous puncture. CSF and blood were centrifuged (after coagulation in case of blood) and aliquoted. CSF and blood samples were immediately snap-frozen in liquid nitrogen and were transferred in liquid nitrogen to the IBB biobank facilities where they were kept frozen at $-80^{\circ} \mathrm{C}$ until subsequent analysis [39].

\section{CSF analysis of AD biomarkers}

CSF biomarkers analysis (P-tau181, T-tau, $\mathrm{A} \beta_{1-42}$ ) was performed at the UAntwerp BIODEM laboratory, using manual ELISA techniques (INNOTEST ${ }^{\circledR} \quad \beta$-Amyloid1-42, $\quad$ INNOTEST ${ }^{\circledR}$ hTau-Ag, and INNOTEST ${ }^{\circledR}$ Phospho-Tau181P, respectively; Fujirebio Europe, Ghent, Belgium) [39].

\section{Melatonin analysis}

Melatonin analysis in CSF and serum was performed by the Chrono@Work laboratory, Groningen, using a liquid chromatography mass spectrometry (LC-MS/MS) method on the Waters ${ }^{\circledR} \mathrm{Xevo}^{\mathrm{TM}} \mathrm{TQ}-$ MS triple quadrupole mass spectrometer with lower limit of quantification of $2.32 \mathrm{pg} / \mathrm{ml}$, inter-assay CV for low levels $(4.99 \mathrm{pg} / \mathrm{ml}): 9.5 \%$, for high levels (69.68 pg/ml): $4.2 \%$, intra-assay CV for low levels $(4.76 \mathrm{pg} / \mathrm{ml}): 8.9 \%$, for high levels $(73.86 \mathrm{pg} / \mathrm{ml})$ : $3.5 \%$ [45].

\section{Statistical analysis}

Analyses were performed using the SPSS ${ }^{2} 27.0$ software package. Values are reported as number, median with interquartile range (IQR), displayed as Q1 and Q3, or mean with standard deviation (SD). Since 13 CSF samples and 20 serum samples had melatonin levels below the limit of quantification (BLOQ) $(<2.3 \mathrm{pg} / \mathrm{ml})$, we performed two separate analyses. In the first analysis we substituted the values BLOQ as follows: [limit of quantification $/ 2]=1.15 \mathrm{pg} / \mathrm{ml}$, while for the second analysis we excluded those values BLOQ.

Based on our sample size calculation with alpha set at 0.05 and beta set at $0.2,11$ patients belonging to the $\mathrm{AD}$ continuum needed to be included to assess the correlation between serum and CSF melatonin values (sample-size.net). Based on the values reported by Sirin et al. [14], and based on our study population distribution, 24 (13/8/3 [ADD/MCI/ healthy controls]) participants needed to be included to assess for differences in melatonin values between these different groups, as calculated with GPower ${ }^{\circledR}$ 3.1. Due to limited sample size and not normally distributed melatonin values as measured by Kolmogorov-Smirnov test, nonparametric tests were applied. To detect differences in categorical binary data between groups, the Fisher-Exact test was used (gender, BEHAVE-AD E score, medication intake, APOE status). Nonparametric tests (Mann-Whitney $\mathrm{U}$ test for pairwise comparisons and Kruskall Wallis test with Bonferroni correction for multiple groups) were applied to assess for differences of continuous data (age, MMSE scores, GDeprS, CSDD, BEHAVE$\mathrm{AD}, \mathrm{MFS}, \mathrm{CMAI}$, melatonin concentrations in CSF and serum). We used Quade's ANCOVA to correct for age in the comparison of CSF and serum melatonin between ADD, MCI and healthy controls. Alpha was set at 0.05 . 


\section{RESULTS}

\section{Patient characteristics}

We included 23 patients with ADD, 13 patients with MCI, and 5 cognitively healthy controls. Demographic and clinical characteristics of participants can be found in Table 1. ADD, MCI, and healthy controls did not differ significantly regarding gender, age, or $A P O E \varepsilon 4$ carrier status. However, AD continuum patients tended to be older than the healthy controls. MMSE scores at inclusion were significantly lower in the ADD patients as compared to the MCI patients and healthy controls $(p<0.001)$ and in the MCI patients as compared to healthy controls $(p=0.003)$.
ADD and MCI patients tended to have a larger yearly MMSE score change decrease than healthy controls, however the differences did not reach significance. The MMSE score change from baseline to year 2 was significantly higher in the ADD patients than in healthy controls $(p=0.042)$. BEHAVE-AD scores, GDeprS, CSDD, MFS, CMAI, intake of anti-AD medication, intake of sleep medication, and intake of antidepressants could only be compared between patients with ADD and patients with MCI because we did not have this information for healthy controls. There were no significant differences between $\mathrm{ADD}$ and MCI patients regarding BEHAVE-AD E score. However, there tended to be more MCI patients than ADD patients with BEHAVE-AD E score of 0

Table 1

Overview of patient characteristics. Data is reported as number or median with IQR

\begin{tabular}{|c|c|c|c|c|}
\hline & $\operatorname{ADD}(n=23)$ & $\operatorname{MCI}(n=13)$ & Healthy controls $(n=5)$ & $p$ \\
\hline $\mathrm{M} / \mathrm{F}$ & $7 / 16$ & $5 / 8$ & $1 / 4$ & 0.801 \\
\hline Age & $77[72.5 ; 80.5]$ & $74[68 ; 77]$ & $70[69 ; 70]$ & $\mathbf{0 . 0 3 8}{ }^{\mathrm{a}}$ \\
\hline MMSE score at baseline & $13[9 ; 16.5]$ & $25[25 ; 26]$ & $29[29 ; 30]$ & $<0.001^{\text {b }}$ \\
\hline Yearly MMSE score change* & $-2[0 ;-4.5]$ & $-1[0.4 ;-1.8]$ & $0[0 ; 0]$ & 0.202 \\
\hline MMSE year 1 - MMSE baseline & $-2[0 ;-4](n=21)$ & $0[-1,75 ; 2](n=10)$ & $0[-0.5 ; 0.5](n=3)$ & 0.137 \\
\hline MMSE year 2 - baseline & $-5[-8 ;-3.75](n=8)$ & $-3[-3 ; 0.5](n=7)$ & $0[0 ; 0.5](n=3)$ & $0.020^{c}$ \\
\hline BEHAVE-AD total score & $10[6 ; 17.5]$ & $3[2 ; 6]$ & - & 0.002 \\
\hline BEHAVE-AD A (/21) & $0[0 ; 3]$ & $0[0 ; 0]$ & - & 0.011 \\
\hline BEHAVE-AD B (/15) & $0[0 ; 0]$ & $0[0 ; 0]$ & - & 0.117 \\
\hline BEHAVE-AD C (/9) & $3[2 ; 5.5]$ & $0[0 ; 0]$ & - & $<0.001$ \\
\hline BEHAVE-AD D (/9) & $3[0.5 ; 5.5]$ & $0[0 ; 1]$ & - & 0.012 \\
\hline BEHAVE-AD E (/3) & $0[0 ; 1.5]$ & $0[0 ; 0]$ & - & 0.121 \\
\hline BEHAVE-AD F (/6) & $1[0 ; 2]$ & $1[0 ; 2]$ & - & 0.780 \\
\hline BEHAVE-AD G (/12) & $0[0 ; 0]$ & $0[0 ; 2]$ & - & 0.359 \\
\hline \multicolumn{5}{|l|}{ Behave-AD E score } \\
\hline 0 & $12[52 \%]$ & $10[77 \%]$ & & 0.175 \\
\hline 1 & $5[22 \%]$ & $2[15 \%]$ & - & 1.000 \\
\hline 2 & $5[22 \%]$ & $1[8 \%]$ & & 0.385 \\
\hline 3 & $1[4 \%]$ & $0[0 \%]$ & & 1.000 \\
\hline Anti-AD medication & 5 & 0 & - & 0.136 \\
\hline Sleep medication & 7 & 4 & - & 1.000 \\
\hline Antidepressants & 7 & 1 & - & 0.213 \\
\hline GDeprS & $9[4 ; 11](n=21)$ & $6[3 ; 8](n=12)$ & - & 0.084 \\
\hline CSDD & $6[4 ; 8.5]$ & $4[1 ; 6]$ & - & 0.094 \\
\hline MFS & $4[3 ; 5]$ & $1.5[0 ; 2]$ & - & $<0.001$ \\
\hline CMAI & $42[33.5 ; 54.5]$ & $31[29 ; 35]$ & - & 0.002 \\
\hline$A P O E \& 4$ carrier status & $(n=22)$ & $(n=10)$ & & \\
\hline no $\varepsilon 4$ alleles & $9[41 \%]$ & $4[40 \%]$ & $1[20 \%]$ & 0.792 \\
\hline$\geq$ one $\varepsilon 4$ allele & $13[59 \%]$ & $6[60 \%]$ & $4[80 \%]$ & 0.792 \\
\hline one $\varepsilon 4$ allele & $10[45 \%]$ & $4[40 \%]$ & $3[60 \%]$ & 0.804 \\
\hline two $\varepsilon 4$ alleles & $3[14 \%]$ & $2[20 \%]$ & 1 [20\%] & 0.843 \\
\hline
\end{tabular}

*Yearly MMSE score changes is defined as "(last MMSE score measured - MMSE score baseline) / years between both". Medication intake was missing in 3 healthy controls and displayed as not available (-) for all healthy controls since $60 \%$ of data for healthy controls was missing. BEHAVE-AD E, GDeprS and CSDD scores were not available (-) for healthy controls. GDeprS was furthermore missing in 2 $\mathrm{ADD}$ and $1 \mathrm{MCI}$ patient. APOE status was missing in $1 \mathrm{ADD}$ and $3 \mathrm{MCI}$ patients. "MMSE year 1 - MSSE baseline" was missing in $2 \mathrm{ADD}$, $3 \mathrm{MCI}$, and 2 healthy controls; "MMSE year 2 - MMSE baseline" in $15 \mathrm{ADD}, 6 \mathrm{MCI}$, and 2 healthy controls. ${ }^{a}$ No significant differences between groups after Bonferroni correction. ${ }^{\mathrm{b}} \mathrm{ADD}$ versus MCI: $p<0.001$; ADD versus healthy controls: $p<0.001$; MCI versus healthy controls: $p=0.003$. c) ADD versus healthy controls: $p=0.042$ after Bonferroni correction, no significant differences between other groups. Patients only took donepezil as anti-AD medication. Sleep medication included chloralhydrate, benzodiazepines, trazodone, and zolpidem. Antidepressants included venlafaxine, sertraline, paroxetine, and trazodone 
[77\% versus 52\%; $p=0.175]$ and more ADD patients than MCI patients with BEHAVE-AD E score of 1-3 [ $48 \%$ versus $23 \% ; p=0.175$ ]. Furthermore, the ADD group tended to score higher on depression scales and tended to have larger intake of antidepressants than the MCI group.

\section{Serum versus CSF daytime melatonin in $A D$ continuum: data BLOQ substituted as $1.15 \mathrm{pg} / \mathrm{ml}$}

Spearman rank correlation coefficient showed a significant positive association between serum and CSF melatonin levels in the AD continuum $(r=0.751 ; p<0.001 ; n=36)$, and in the ADD group $(r=0.669 ; p<0.001 ; n=23)$ and in the MCI group separately $(r=0.671 ; p=0.012 ; n=13)$. The scatterplot for CSF and serum melatonin in the $\mathrm{AD}$ continuum is shown in Fig. 1. In case of healthy controls, there is also a significant positive association between CSF and serum melatonin levels ( $r=0.894$; $p=0.041 ; n=5)$.

Since depression and altered day-night rhythms might influence daytime melatonin levels, we assessed whether the correlation coefficient changed after removal of those patients with significant depressive symptoms suggestive of major depression (CSDD or GDeprS $>11 ; n=8$ ) and after removing those with day/night disturbances (BEHAVE AD $\mathrm{E}>1 ; n=7)$. After removing patients with major

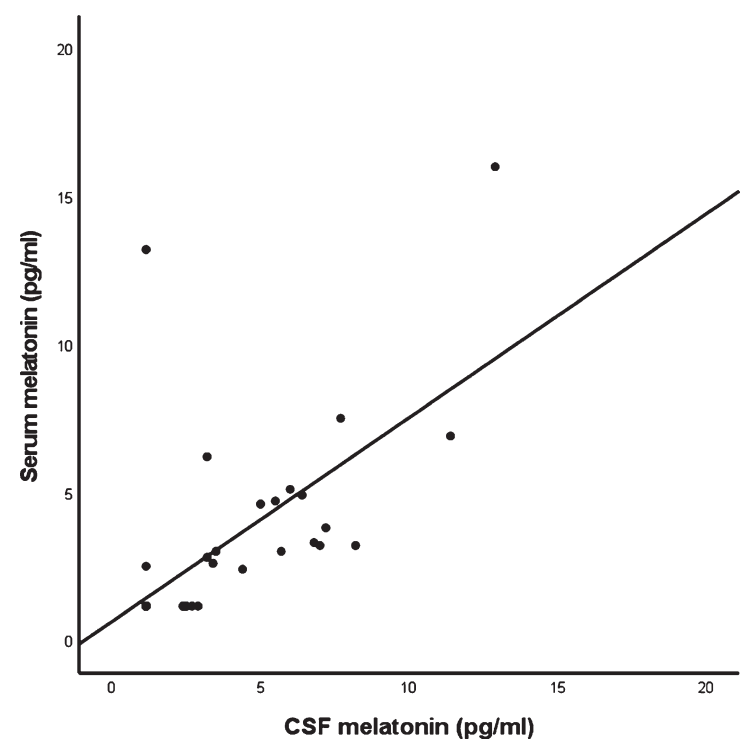

Fig. 1. Scatterplot of CSF melatonin versus serum melatonin in the $\mathrm{AD}$ continuum $(n=36)$ with BLOQ values substituted as 1.15 $\mathrm{pg} / \mathrm{ml}$. depressive complaints, the association remained significant $(r=0.715, p<0.001 ; n=28)$. The same was seen after removal of the patients with significant depressive symptoms and with intake of antidepressants $(n=12)(r=0.657 ; p<0.001 ; n=24)$ or after removal of the patients with day/night disturbances $(r=0.707 ; p<0.001 ; n=29)$. Even after removal of 1 participant with complete reversal of day-night rhythm (BEHAVE AD E $=3 ; n=1$ ), the correlation coefficient remained unchanged $(r=0.749 ; p<0.001$; $n=35$ ). The effects of sleep medication (chloralhydrate, benzodiazepines, trazodone, and zolpidem) and antidepressants (venlafaxine, sertraline, paroxetine, and trazodone) intake were also assessed separately: correlation did not increase after removal of patients with intake of sleep medication $(n=11)$ or antidepressants $(n=8)(r=0.622 ; p<0.001 ; n=25$ and $r=0.708 ; p<0.001 ; n=28$ respectively).

\section{Serum versus $C S F$ daytime melatonin in the $A D$ continuum: data $B L O Q$ disregarded}

When disregarding melatonin levels BLOQ, the correlation coefficient was lower than after substitution of melatonin levels BLOQ but remained significant in the $\mathrm{AD}$ continuum $(r=0.543 ; p=0.024$; $n=17$ ). However, after removing patients with significant depressive complaints (CSDD or GDeprS $>11$, $n=4$ ) or patients with significant depressive symptoms and antidepressant intake $(n=7)$, correlation coefficient dropped and did not remain significant $(r=0.482 ; p=0.095 ; n=13$ and $r=0.249 ; p=0.487$;

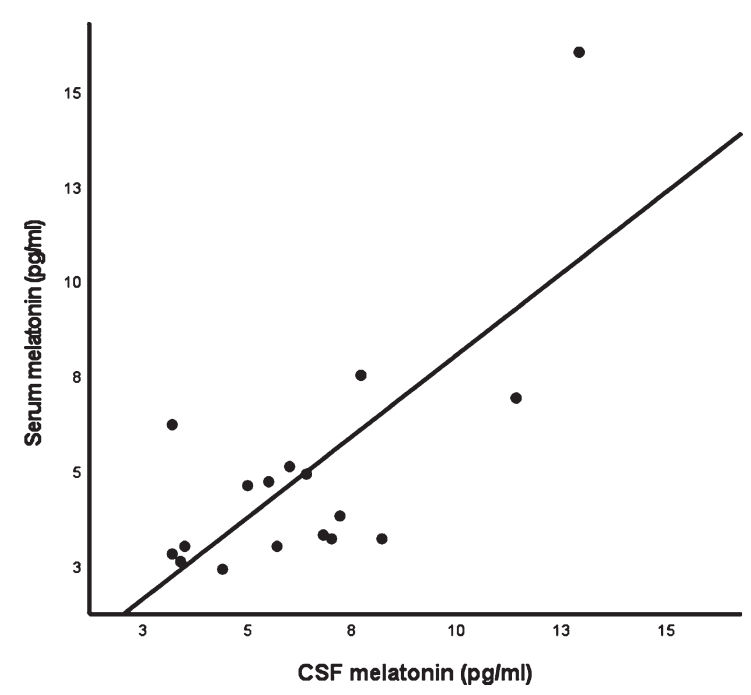

Fig. 2. Scatterplot of CSF melatonin versus serum melatonin in the $\mathrm{AD}$ continuum $(n=17)$ when BLOQ values were disregarded. 
$n=10)$. There were no significant differences in CSF and serum melatonin values between depressed and non-depressed AD patients $(p=0.367 ; p=0.271$ respectively). On the other hand, after removal of patients with diurnal rhythm disturbances (BEHAVE AD E $>1 ; n=5$ ), the correlation coefficient increased $(r=0.647 ; p=0.023 ; n=12)$. After removal of 1 participant with complete reversal of day-night rhythm (BEHAVE AD E=3), the correlation coefficient remained unchanged $(r=0.575 ; p=0.020 ; n=16)$. The scatterplot for CSF and serum melatonin in the AD continuum with values BLOQ disregarded is shown in Fig. 2.

\section{Melatonin levels and cognition}

Mean +/- SD CSF and serum melatonin values in the ADD, MCI, and healthy control group are reported in Table 2 (BLOQ values substituted as $1.15 \mathrm{pg} / \mathrm{ml}$ ) and Table 3 (BLOQ values disregarded). No statistical differences were found regarding CSF and serum melatonin between ADD, MCI and healthy controls. When correcting for age, differences regarding CSF and serum melatonin between these three groups remained insignificant with BLOQ substituted $(p=0.588 ; p=0.334)$ and with BLOQ disregarded $(p=0.566 ; p=0.482)$. There were no GDeprS or CSDD scores available for the healthy controls. However, we knew that healthy controls did not have major depressive complaints, so we did a re-analysis for comparison of CSF and serum melatonin levels between ADD, MCI and healthy controls without depressive complaints (GDeprs or CSDD > 11). Differences remained insignificant $(p=0.767 ; p=0.258$ for CSF and serum melatonin respectively with substitution of values BLOQ; $p=0.171$ and $p=0.371$ without substitution).
Furthermore, we evaluated the link between daytime melatonin levels in the $\mathrm{AD}$ continuum and cognition by correlating melatonin levels with MMSE scores at baseline and yearly MMSE score change. No significant correlations were found between CSF or serum melatonin levels and MMSE at baseline $(r=-0.226 ; p=0.185$ and $r=-0.100$; $p=0.561$ for CSF and serum with substitution of values BLOQ respectively; $r=-0.155 ; p=0.471$; $r=-0.023 ; p=0.926$ for CSF and serum respectively without substitution of values BLOQ), nor between CSF or serum melatonin and MMSE score change ( $r=-0.092 ; p=0.596$ and $r=-0.013$, $p=0.938$ for CSF and serum with substitution of values BLOQ respectively; $r=0.002 ; p=0.993$ and $r=0.376 ; p=0.112$ for CSF and serum respectively without substitution of values BLOQ).

\section{Melatonin levels and $A \beta_{1-42}, P$-tau181, and T-tau}

We also evaluated the association between melatonin levels and CSF $A \beta_{1-42}$, P-tau181, and T-tau levels in the $\mathrm{AD}$ continuum but could not find any correlation $(r=-0.059 ; p=0.730, r=-0.237$; $p=0.171, r=-0.181 ; p=0.297$ for $\mathrm{CSF} \mathrm{A} \beta_{1-42}$, P-tau181, and T-tau and CSF melatonin with substitution of values BLOQ respectively; $r=0.123$; $p=0.474, r=-0.124 ; p=0.480, r=-0.204 ; p=0.241$ for CSF A $\beta_{1-42}$, P-tau181, and T-tau and serum melatonin with substitution of values BLOQ respectively; $r=0.059 ; p=0.786, r=-0.240 ; p=0.271, r=-0.152$; $p=0.489$ for CSF $\mathrm{A} \beta_{1-42}, \mathrm{P}-\operatorname{tau} 181$, and $\mathrm{T}$-tau and CSF melatonin without substitution of values BLOQ respectively; $r=0.294 ; p=0.222 ; r=-0.028$; $p=0.914 ; r=-0.076 ; p=0.758$ for $\mathrm{CSF} \mathrm{A} \beta_{1-42}$, P-tau181, and T-tau and serum melatonin without substitution of values BLOQ respectively).

Table 2

Mean +/- SD CSF and serum melatonin levels in patients with ADD and MCI and in healthy controls with values BLOQ substituted as $1.15 \mathrm{pg} / \mathrm{ml}$

\begin{tabular}{lcccc}
\hline & ADD $(n=23)$ & MCI $(n=13)$ & Healthy controls $(n=5)$ & $p$ \\
\hline CSF melatonin $[\mathrm{pg} / \mathrm{ml}]$ & $4.09 \pm 2.83$ & $3.46 \pm 3.45$ & $3.25 \pm 1.41$ & 0.578 \\
Serum melatonin $[\mathrm{pg} / \mathrm{ml}]$ & $3.57 \pm 2.92$ & $2.79 \pm 4.05$ & $2.83 \pm 3.15$ & 0.225 \\
\hline
\end{tabular}

Table 3

Mean +/- SD CSF and serum melatonin levels in patients with ADD and MCI and in healthy controls without values BLOQ

\begin{tabular}{lcccc}
\hline & ADD & MCI & Healthy controls & $p$ \\
\hline CSF melatonin $[\mathrm{pg} / \mathrm{ml}]$ & $5.38 \pm 2.44(n=16)$ & $4.90 \pm 3.77(n=8)$ & $3.78 \pm 0.90(n=4)$ & 0.455 \\
Serum melatonin $[\mathrm{pg} / \mathrm{ml}]$ & $5.13 \pm 2.78(n=14)$ & $5.42 \pm 5.92(n=5)$ & $5.35 \pm 4.31(n=2)$ & 0.461 \\
\hline
\end{tabular}




\section{Melatonin levels and $A P O E$}

As shown in Table 1, there were no statistical differences in $\varepsilon 4$ carrier status between ADD, MCI patients, and healthy controls. Furthermore, no statistical differences could be found in CSF and serum melatonin levels comparing patients belonging to the $\mathrm{AD}$ continuum carrying two $A P O E$ $\varepsilon 4$ alleles versus those who do not carry an $\varepsilon 4$ allele $(p=0.960 ; p=0.797$ with substitution of values BLOQ; $p=0.397 ; p=0.909$ without substitution of values BLOQ), nor regarding those who carry one $\varepsilon 4$ allele versus those not carrying $\varepsilon 4$ alleles ( $p=0.590 ; p=0.979$ with substitution; $p=0.649$; $p=0.701$ without substitution), nor when comparing those carrying two $\varepsilon 4$ alleles versus those carrying one $\varepsilon 4$ allele ( $p=0.925 ; p=0.732$ with substitution; $p=0.610 ; p=1.000$ without substitution).

\section{Melatonin levels and BEHAVE-AD E scores}

We compared CSF and blood melatonin values between those $\mathrm{AD}$ continuum patients with BEHAVE-AD E score $\leq 1$ and $>1$. We could not find any significant differences in CSF or blood melatonin levels when values BLOQ were substituted $(p=0.528, p=0.364$ for CSF and blood respectively), nor when values BLOQ were not substituted $(p=0.499, p=0.853$ for CSF and blood respectively). We could neither find a correlation between BEHAVE-AD E score and CSF/serum melatonin values when values BLOQ were substituted $(r=0.128 ; p=0.458$ for CSF and $r=0.134, p=0.435$ for serum) and when values BLOQ were not substituted $(r=0.125 ; p=0.561$ for CSF and $r=0.086$; $p=0.727$ for serum) in the $\mathrm{AD}$ continuum.

\section{DISCUSSION}

Melatonin disruptions have been widely described in $\mathrm{AD}$ patients. SCN cell number and volume as well as functional pineal gland volume decreases in $\mathrm{AD}$, with disrupted pineal gland melatonin production and alterations in ventricular CSF and blood melatonin levels as a consequence $[9,11-13,16$, $17,19,25]$. Ventricular CSF melatonin levels correlate with pineal gland melatonin in a pathologically confirmed AD cohort [23], whereas the correlation between serum and spinal CSF melatonin concentrations has only been described in 13 healthy volunteers [6]. In the present study, we found that daytime serum melatonin levels could accurately reflect daytime spinal CSF melatonin levels in the AD continuum as well, with a correlation coefficient similar to that found in healthy volunteers when melatonin levels BLOQ were substituted [6] $(r=0.751$ here versus $r=0.72$ in healthy volunteers), suggesting valid use of blood melatonin levels to study melatonin disruptions in AD during the day. This entails an advantage since venipuncture is less invasive than lumbar puncture. Furthermore, multiple blood samples can be taken with minimal stress, giving the potential to assess changes in melatonin levels during a $24 \mathrm{~h}$ period and venipuncture can easier be done in dark environment than lumbar puncture, making it easier to assess night-time melatonin levels although accurate correlation between the much higher nighttime blood and CSF melatonin levels remains to be confirmed in a prospective AD study. Presence of depressive complaints, sleeping disturbances, and intake of antidepressants or sleep medication did not seem to negatively influence the positive correlation between CSF and serum melatonin when melatonin values BLOQ were substituted. However, when melatonin values BLOQ were disregarded, the correlation coefficient decreased after removal of depressive patients but increased after removal of patients with day/night disturbances. Since there were no significant differences in melatonin values between depressed AD patients versus non-depressed $\mathrm{AD}$ patients, we hypothesize that this drop might be due to the low sample size remaining after removal of depressed patients. Sleeping disturbances might have a negative effect on the correlation coefficient which has already been demonstrated in healthy persons [36]. However, even with inclusion of patients with sleeping disturbances in this study, the correlation remained significant. Overall, we conclude that daytime blood melatonin reflects daytime spinal CSF melatonin in the AD continuum.

Current literature suggests a significant decrease in nocturnal blood melatonin production in $\mathrm{AD}$ patients $[11,12,15,24,25,46,47]$. Furthermore, a decrease in total daytime production [15], advanced chronophases [12, 13], and wider scatters of the acrophase [11] have been described. However, regarding daytime melatonin levels, literature is inconclusive [16]. Increased daytime blood melatonin levels in patients with dementia and ADD as compared to age-matched controls have been reported [12, 13], whereas others describe decreased daytime blood melatonin levels as compared to healthy controls [14, 26, 27], with blood sampling 
done between 08:00-09:00 AM [14], 07:00-08:00 AM [26], and at 12:00 AM [27] respectively. Souêtre et al. [48] and Zverova et al. [28] could not find a statistically significant difference in daytime blood melatonin levels between demented patients and healthy elderly. As reported in the systematic review by Nous et al. [16], the paper by Zverova et al. [28] is the most recent paper regarding daytime blood melatonin levels. Our findings are in line with their findings. We could not find a statistically significant difference regarding daytime melatonin levels between patients with ADD, MCI, and healthy controls, not even when correcting for age, or, after removal of all patients with depressive symptoms. The fact that Sirin et al. [14] and Ozcankaya et al. [27] found decreased blood melatonin levels in ADD patients as compared to controls could possibly be explained by their early blood sampling: ADD patients might have advanced chronophases [12, 13], possibly contributing to an earlier drop in melatonin levels in the morning as compared to the healthy controls. However, sampling by Souêtre et al. [48] and Zverova et al. [28] was also performed at 08:00 AM or between 07:00 and 08:00 AM, arguing against previously mentioned argument. Blood and CSF sampling in our study was done between 08:00 AM and 04:00 PM (78\% of sampling was performed between 08:00 AM and 10:00 AM). In this timeframe, we know that plasma melatonin is not tended toward a large degree in variation/shift in healthy controls [5].

Sirin et al. found a correlation between MMSE scores and daytime melatonin levels [14], which we could not confirm. Neither could we find any correlation between daytime melatonin levels and longitudinal MMSE score changes. We hypothesize that daytime melatonin levels do not contribute enough to total melatonin levels. Therefore daytime melatonin levels might not entail positive effects on cognition in $\mathrm{AD}$. On the other hand, we suggest that disruptions in night-time melatonin production, and as such probably also in the total melatonin production, might be more predictive of cognitive decline in AD. Ferrari et al. found a correlation between nighttime melatonin levels and MMSE scores in demented patients [11]. During night-time, melatonin levels increase, making it a much larger contributor to total melatonin production in humans and possible beneficial effects on $\mathrm{AD}$ symptoms than daytime melatonin levels. The link between nocturnal melatonin production in the AD continuum and longitudinal MMSE score changes should therefore be further investigated.
Liu et al. found ventricular CSF melatonin levels to be significantly reduced in patients carrying two $A P O E \varepsilon 4$ alleles when compared to patients carrying an $\varepsilon 3$ and $\varepsilon 4$ allele [9]. In contrast with this, Zverova et al. found that plasma melatonin levels were significantly higher in a group of AD patients who carried at least one $\varepsilon 4$ allele versus those who did not carry an $\varepsilon 4$ allele [28]. We could not find any differences in CSF or serum melatonin concentrations when comparing $\mathrm{AD}$ patients expressing one or two $\varepsilon 4$ alleles as compared to those who did not. The fact that we could not find any differences in $A P O E \varepsilon 4$ carrier status between our AD patients and healthy controls might be explained by the accidentally high prevalence of $A P O E \varepsilon 4$ carrier status in our healthy control group (80\%), which is normally only present in $23.1 \%$ of a Caucasian population [49]. Only one healthy control carrying one $\varepsilon 4$ allele had subjective memory complaints, whereas all other healthy controls had no memory complaints.

Limitations of our study are the retrospective nature of the study, with availability of serum and CSF only taken during the daytime. The fact that sampling was performed during the day, led to melatonin values below the limit of quantification in part of the participants. We tried to address this issue by performing two separate analyses: one where values were substituted as [limit of quantification/2] and one analysis where they were disregarded. Values reported in our study are rather low in comparison with other studies $( \pm 9 \mathrm{pg} / \mathrm{ml}$ Sirin et al. [14], $170 \mathrm{pg} / \mathrm{ml}$ Ozcankaya et al. [27], $34 \mathrm{pg} / \mathrm{ml}$ Zverova et al. [28] for AD patients). However, according to the recent review by Kennaway, melatonin levels in plasma are expected to be less than $5 \mathrm{pg} / \mathrm{ml}$ during the daytime when highquality assay procedures are used, which makes us conclude that measured melatonin values in our study are accurate [1]. Further limitations include the relatively small sample size that however meets our sample size calculation, and the fact that CSF and serum samples from $\mathrm{AD}$ patients were older than those from healthy controls. However, melatonin has been shown to remain stable over time in propylene glycol, glycofurol, and dimethyl sulfoxide at $25^{\circ} \mathrm{C}$ [50], as well as in aqueous solution at $4^{\circ} \mathrm{C}$ and $-70^{\circ} \mathrm{C}$ [51]. Furthermore, our samples were stored at $-80^{\circ} \mathrm{C}$ and had undergone maximally 3 freeze-thaw cycles and it has been shown before that plasma melatonin levels remained unchanged following three freezethaw cycles [52].

Our study also exhibits strengths. We used a good analytical method (LC-MS/MS) for melatonin anal- 
ysis as mass spectrometry provides us with target values for daytime melatonin levels that immunoassays still need to achieve [3]. Furthermore, we used core AD CSF biomarkers for clinical diagnostic categorization (ADD, MCI, and healthy controls) of participants which has not been done before in $\mathrm{AD}$ melatonin studies. As mentioned before, blood and CSF sampling between 08:00 AM and 04:00 PM would not lead to a large degree in variation/shift in melatonin levels, as shown in healthy volunteers' plasma before [5]. Melatonin has been measured in serum and plasma $[14,27,28,36]$. In contrast to melatonin's precursor serotonin, which is stored in platelets requiring serotonin analysis in whole blood or platelet-rich plasma [53], melatonin does not seem to be stored in platelets leading to accurate melatonin measurement in serum and platelet-poor plasma [54]. A high correlation between serum and plasma melatonin has been found in blood samples drawn from 20 Caucasian men $(r=0.97)$ [55].

Larger, prospective clinical studies are needed to detect possible differences in night-time melatonin levels between ADD patients, MCI patients, and healthy controls and to investigate the possible correlations with cognitive decline, as mentioned above, in order to establish further evidence for the use of melatonin as a future treatment option in AD. By using longitudinal neuropsychological follow-up, new prospective studies would enable us to study the correlation between night-time melatonin values and cognitive decline in AD. Prospective studies entail the availability to standardize sampling at the same time of day in different participants, and to correct for environmental influences such as light, caffeine intake, and tryptophan-enriched food intake in particular, since melatonin is formed out of serotonin.

\section{CONCLUSION}

Serum daytime melatonin levels accurately reflect daytime CSF melatonin levels in the AD continuum, expressing the possibility to use blood sampling when studying melatonin's disruptions and effects in AD. However, the correlation between night-time blood and CSF melatonin values remains to be proven. The sole need of blood for melatonin measurement in $\mathrm{AD}$ would bring along several advantages, because venipuncture is less invasive and easier performed in dark environment than lumbar puncture. Furthermore, there is a possibility to collect multiple blood samples within a $24-\mathrm{h}$ period. We conclude from our retrospective study that daytime melatonin levels are not associated with cognitive status (ADD versus $\mathrm{MCI}$ due to $\mathrm{AD}$ versus healthy controls), nor can these levels predict cognitive decline. We hypothesize that there might be a more important role for night-time melatonin levels in the reflection and prediction of cognitive decline in AD.

\section{ACKNOWLEDGMENTS}

This work was supported by Fonds Wetenschappelijk Onderzoek (FWO), Flanders, grant number B040419N and Vrije Universtiteit Brussel (VUB) OZR Back-up Mandate (OZR3629). The funders had no role in study design, data collection, data analysis or interpretation of the data, nor in the writing of the report or decision to submit the article for publication. Published with support of 'Universitaire Stichting' of Belgium.

Authors' disclosures available online (https:// www.j-alz.com/manuscript-disclosures/21-0562r1).

\section{REFERENCES}

[1] Kennaway DJ (2020) Measuring melatonin by immunoassay. J Pineal Res 69, e12657.

[2] Grivas TB, Savvidou OD (2007) Melatonin the "light of night" in human biology and adolescent idiopathic scoliosis. Scoliosis 2, 6.

[3] Kennaway DJ (2019) A critical review of melatonin assays: Past and present. J Pineal Res 67, e12572.

[4] Vincent B (2018) Protective roles of melatonin against the amyloid-dependent development of Alzheimer's disease: A critical review. Pharmacol Res 134, 223-237.

[5] Janssens J, Atmosoerodjo SD, Vermeiren Y, Absalom AR, den Daas I, De Deyn PP (2019) Sampling issues of cerebrospinal fluid and plasma monoamines: Investigation of the circadian rhythm and rostrocaudal concentration gradient. Neurochem Int 128, 154-162.

[6] Rousseau A, Petren S, Plannthin J, Eklundh T, Nordin C (1999) Serum and cerebrospinal fluid concentrations of melatonin: A pilot study in healthy male volunteers. $\mathrm{J} \mathrm{Neu}$ ral Transm 106, 883-888.

[7] Tan D, Manchester LC, Sanchez-barcelo E, Mediavilla MD, Reiter RJ (2010) Significance of high levels of endogenous melatonin in mammalian cerebrospinal fluid and in the central nervous system. Curr Neuropharmacol 8, 162-167.

[8] Brzezinski A (1997) Melatonin in humans. N Engl J Med 336, 186-195.

[9] Liu RY, Zhou JN, van Heerikhuize J, Hofman MA, Swaab DF (1999) Decreased melatonin levels in postmortem cerebrospinal fluid in relation to aging, Alzheimer's disease, and apolipoprotein E-epsilon4/4 genotype. J Clin Endocrinol Metab 84, 323-327.

[10] Skene DJ, Vivien-Roels B, Sparks DL, Hunsaker JC, Pevet P, Ravid D, Swaab DF (1990) Daily variation in the concentration of melatonin and 5-methoxytryptophol in the human pineal gland: Effect of age and Alzheimer's disease. Brain Res 528, 170-174. 
[11] Ferrari E, Arcaini A, Gornati R, Pelanconi L, Cravello L, Fioravanti M, Solerte SB, Magri F (2000) Pineal and pituitary-adrenocortical function in physiological aging and in senile dementia. Exp Gerontol 35, 1239-1250.

[12] Ohashi Y, Okamoto N, Uchida K, Iyo M, Mori N, Morita Y (1999) Daily rhythm of serum melatonin levels and effect of light exposure in patients with dementia of the Alzheimer's type. Biol Psychiatry 45, 1646-1652.

[13] Uchida K, Okamoto N, Ohara K, Morita Y (1996) Daily rhythm of serum melatonin in patients with dementia of the degenerate type. Brain Res 717, 154-159.

[14] Şİİ́n FB, Doğuç DK, Vural H, Eren İ, İnanli İ, Sütçü R, Delİbaş N (2015) Plasma 8-isoPGF2 $\alpha$ and serum melatonin levels in patients with minimal cognitive impairment and Alzheimer disease. Turk J Med Sci 45, 1073-1077.

[15] Mishima K, Tozawa T, Satoh K, Matsumoto Y, Hishikawa Y, Okawa M (1999) Melatonin secretion rhythm disorders in patients with senile dementia of Alzheimer's type with disturbed sleep - waking. Biol Psychiatry 45, 417-421.

[16] Nous A, Engelborghs S, Smolders I (2021) Melatonin levels in the Alzheimer's disease continuum: A systematic review. Alzheimers Res Ther 13, 52.

[17] Mahlberg R, Walther S, Kalus P, Bohner G, Haedel S, Reischies FM, Kühl K-P, Hellweg R, Kunz D (2008) Pineal calcification in Alzheimer's disease: An in vivo study using computed tomography. Neurobiol Aging 29, 203-209.

[18] Matsuoka T, Imai A, Fujimoto H, Kato Y, Shibata K, Nakamura K, Yokota H, Yamada K, Narumoto J (2018) Reduced pineal volume in alzheimer disease: A retrospective cross-sectional MR imaging study. Radiology 286, 239-248.

[19] Swaab DF, Roozendaal B, Ravid R, Velis DN, Gooren L, Williams RS (1987) Suprachiasmatic nucleus in aging, Alzheimer's disease, transsexuality and Prader-Willi syndrome. Prog Brain Res 72, 301-310.

[20] Stopa EG, Volicer L, Kuo-Leblanc V, Harper D, Lathi D, Tate B, Satlin A (1999) Pathologic evaluation of the human suprachiasmatic nucleus in severe dementia. J Neuropathol Exp Neurol 58, 29-39.

[21] Harper DG, Stopa EG, Kuo-Leblanc V, McKee AC, Asayama K, Volicer L, Kowall N, Satlin A (2008) Dorsomedial SCN neuronal subpopulations subserve different functions in human dementia. Brain 131, 1609-1617.

[22] Zhou JN, Hofman MA, Swaab DF (1995) VIP neurons in the human SCN in relation to sex, age, and Alzheimer's disease. Neurobiol Aging 16, 571-576.

[23] Wu Y-H, Feenstra MGP, Zhou J-N, Liu R-Y, Torano JS, Van Kan HJM, Fischer DF, Ravid R, Swaab DF (2003) Molecular changes underlying reduced pineal melatonin levels in Alzheimer disease: Alterations in preclinical and clinical stages. J Clin Endocrinol Metab 88, 5898-5906.

[24] Magri F, Locatelli M, Balza G, Molla G, Cuzzoni G, Fioravanti M, Solerte SB, Ferrari E (1997) Changes in endocrine circadian rhythms as markers of physiological and pathological brain aging. Chronobiol Int 14, 385-396.

[25] Dori D, Casale G, Solerte SB, Fioravanti M, Migliorati G, Cuzzoni G, Ferrari E (1994) Chrono-neuroendocrinological aspects of physiological aging and senile dementia. Chronobiologia 21, 121-126.

[26] Mishima K, Okawa M, Hishikawa Y, Hozumi S, Hori H, Takahashi K (1994) Morning bright light therapy for sleep and behavior disorders in elderly patients with dementia. Acta Psychiatr Scand 89, 1-7.

[27] Ozcankaya R, Delibas N (2002) Malondialdehyde, superoxide dismutase, melatonin, iron, copper, and zinc blood concentrations in patients with Alzheimer disease: Crosssectional study. Croat Med J 43, 28-32.

[28] Zverova M, Kitzlerova E, Fisar Z, Jirak R, Hroudova J, Benakova H, Lelkova P, Martasek P, Raboch J (2018) Interplay between the APOE genotype and possible plasma biomarkers in Alzheimer's disease. Curr Alzheimer Res 15, 938-950.

[29] Liu Y-J, Meng F-T, Wang L-L, Zhang L-F, Cheng X-P, Zhou J-N (2012) Apolipoprotein E influences melatonin biosynthesis by regulating NAT and MAOA expression in C6 cells. $J$ Pineal Res 52, 397-402.

[30] Iliff JJ, Wang M, Liao Y, Plogg BA, Peng W, Gundersen GA, Benveniste H, Vates GE, Deane R, Goldman SA, Nagelhus EA, Nedergaard M (2012) A paravascular pathway facilitates CSF flow through the brain parenchyma and the clearance of interstitial solutes, including amyloid $\beta$. Sci Transl Med 4, 147ra111.

[31] Jessen NA, Munk ASF, Lundgaard I, Nedergaard M (2015) The glymphatic system: A beginner's guide. Neurochem Res 40, 2583-2599.

[32] Spinedi E, Cardinali DP (2019) Neuroendocrine-metabolic dysfunction and sleep disturbances in neurodegenerative disorders: Focus on Alzheimer's disease and melatonin. Neuroendocrinology 108, 354-364.

[33] Cardinali DP, Vigo DE, Olivar N, Vidal MF, Furio AM, Brusco LI (2012) Therapeutic application of melatonin in mild cognitive impairment. Am J Neurodegener Dis 1, 280-291.

[34] Furio AM, Brusco LI, Cardinale DP (2007) Possible therapeutic value of melatonin in mild cognitive impairment: A retrospective study. J Pineal Res 43, 404-409.

[35] Wang Y, Zheng W, Ng CH, Ungvari GS, Wei W, Xiang Y (2017) Meta-analysis of randomised, double-blind, placebo controlled trials of melatonin in Alzheimer's disease. Int $J$ Geriatr Psychiatry 32, 50-57.

[36] Bumb JM, Schwarz E, Enning F, Rohleder C, Findeisen P, Leweke FM, Noelte I (2014) Sleep deprivation in humans: Effects on melatonin in cerebrospinal fluid and serum. Sleep Biol Rhythms 12, 69-72.

[37] Van der Mussele S, Marien P, Saerens J, Somers N, Goeman J, De Deyn PP, Engelborghs S (2014) Behavioral syndromes in mild cognitive impairment and Alzheimer's disease. $J$ Alzheimers Dis 38, 319-329.

[38] Van der Mussele S, Marien P, Saerens J, Somers N, Goeman J, De Deyn PP, Engelborghs S (2015) Psychosis associated behavioral and psychological signs and symptoms in mild cognitive impairment and Alzheimer's dementia. Aging Ment Health 19, 818-828.

[39] Somers C, Struyfs H, Goossens J, Niemantsverdriet E, Luyckx J, De Roeck N, De Roeck E, De Vil B, Cras P, Martin J-J, De Deyn P-P, Bjerke M, Engelborghs S (2016) A decade of cerebrospinal fluid biomarkers for Alzheimer's disease in Belgium. J Alzheimers Dis 54, 383-395.

[40] Mckhann GM, Knopman DS, Chertkow H, Hyman BT, Jack CR, Kawas CH, Klunk WE, Koroshetz WJ, Manly JJ, Mayeux R, Mohs RC, Morris JC, Rossor MN, Scheltens P, Carrillo MC, Thies B, Weintraub S, Phelps $\mathrm{CH}$ (2011) The diagnosis of dementia due to Alzheimer's disease: Recommendations from the National Institute on Aging-Alzheimer's Association workgroups on diagnostic guidelines for Alzheimer's disease. Alzheimers Dement 7, 263-269.

[41] Albert MS, DeKosky ST, Dickson D, Dubois B, Feldman HH, Fox NC, Gamst A, Holtzman DM, Jagust WJ, Petersen 
RC, Snyder PJ, Carrillo MC, Thies B, Phelps CH (2011) The diagnosis of mild cognitive impairment due to Alzheimer's disease: Recommendations from the National Institute on Aging-Alzheimer's Association workgroups on diagnostic guidelines for Alzheimer's disease. Alzheimers Dement 7, 270-279.

[42] Alexopoulos GS, Abrams RC, Young RC, Shamoian CA (1988) Cornell Scale for Depression in Dementia. Biol Psychiatry 23, 271-284.

[43] Yesavage JA (1988) Geriatric Depression Scale. Psychopharmacol Bull 24, 709-711.

[44] Debruyne H, Van Buggenhout M, Le Bastard N, Aries M, Audenaert K, De Deyn PP, Engelborghs S (2009) Is the geriatric depression scale a reliable screening tool for depressive symptoms in elderly patients with cognitive impairment? Int J Geriatr Psychiatry 24, 556-562.

[45] van Faassen M, Bischoff R, Kema IP (2017) Relationship between plasma and salivary melatonin and cortisol investigated by LC-MS/MS. Clin Chem Lab Med 55, 1340-1348.

[46] Ferrari E, Fioravanti M, Magri F, Solerte SB (2000) Variability of interactions between neuroendocrine and immunological functions in physiological aging and dementia of the Alzheimer's type. Ann N Y Acad Sci 917, 582-596.

[47] Nair NP V, Schwartz G, Kin N, Thakur M, Thavundayil JX (1998) Melatonin and cortisol circadian rhythms in Alzheimer's disease patients and normal elderly subjects. In Biological Clocks: Mechanisms and Applications, Touitou, Y, ed., pp. 357-360.
[48] Souêtre E, Salvati E, Krebs B, Belugou JL, Darcourt G (1989) Abnormal melatonin response to 5-methoxypsoralen in dementia. Am J Psychiatry 146, 1037-1040.

[49] Farrer LA, Cupples LA, Haines JL, Hyman B, Kukull WA, Mayeux R, Myers RH, Pericak-Vance MA, Risch N, van Duijn CM (1997) Effects of age, sex, and ethnicity on the association between apolipoprotein E genotype and Alzheimer disease. A meta-analysis. APOE and Alzheimer Disease Meta Analysis Consortium. JAMA 278, 1349-1356.

[50] Zetner D, Rosenberg J (2020) Solubility and stability of melatonin in propylene glycol, glycofurol, and dimethyl sulfoxide. F1000Research 9, 85.

[51] Cavallo A, Hassan M (1995) Stability of melatonin in aqueous solution. J Pineal Res 18, 90-92.

[52] Graham C, Cook MR, Kavet R, Sastre A, Smith DK (1998) Prediction of nocturnal plasma melatonin from morning urinary measures. J Pineal Res 24, 230-238.

[53] Brand T, Anderson GM (2011) The measurement of platelet-poor plasma serotonin: A systematic review of prior reports and recommendations for improved analysis. Clin Chem 57, 1376-1386.

[54] van Faassen M, Peters MAM, Walenkamp AM, de Vries EGE, Oosting SF, Kema IP (2019) Melatonin is not stored in platelets. Clin Chim Acta 498, 17-20.

[55] Hsing AW, Meyer TE, Niwa S, Quraishi SM, Chu LW (2010) Measuring serum melatonin in epidemiologic studies. Cancer Epidemiol Biomarkers Prev 19, 932-937. 